\title{
Penser le nécessaire dialogue des sciences des textes
}

Jean-Michel Adam

\section{Q OpenEdition}

1 Journals

Édition électronique

URL : http://journals.openedition.org/edl/837

DOI : $10.4000 /$ edl. 837

ISSN : 2296-5084

Éditeur

Université de Lausanne

\section{Édition imprimée}

Date de publication : 15 mai 2015

Pagination : 211-236

ISBN : 978-2-940331-45-1

ISSN : 0014-2026

Référence électronique

Jean-Michel Adam, «Penser le nécessaire dialogue des sciences des textes », Études de lettres [En ligne], 1-2 | 2015, mis en ligne le 15 mai 2018, consulté le 19 décembre 2020. URL : http:// journals.openedition.org/edl/837; DOI : https://doi.org/10.4000/edl.837 


\section{PENSER LE NÉCESSAIRE DIALOGUE DES SCIENCES DES TEXTES}

Entre «récit de vie» et «dernière séance», les caractéristiques du genre de la "Leçon d'adieu", même rebaptisée "Leçon d'honneur " ${ }^{1}$, restent vagues, mais il y a dans ce rituel quelque chose qui dépasse nos personnes et nos émotions en faisant sens institutionnellement.

Je prends publiquement congé d'une fonction dont la responsabilité m’a été temporairement confiée, en 1984, pour qu'un autre me remplace et fasse vivre à son tour, avec une nouvelle dynamique, une unité d'enseignement et de recherche qui, grâce au travail des enseignants et chercheurs qui m'ont accompagné tout au long de ces années, a conquis progressivement une identité et une réputation scientifiques. Ce moment est donc d'abord un passage public de témoin au nouveau professeur de linguistique française, Gilles Philippe, auquel je souhaite succès et bonheur dans sa nouvelle fonction. Je ne quitte toutefois pas mes fonctions sans inquiétudes quand je vois le conseiller fédéral radical Johann Schneider-Ammann établir, au nom d'une conception pyramidale ${ }^{2} \mathrm{de}$ la société suisse, un lien entre le nombre de maturités et le chômage en

I. Ce texte est la version préparée pour être prononcée le 15 novembre 2012. Une version plus développée et moins circonstancielle est parue dans un volume d'hommage coordonné par Michèle Monte et Gilles Philippe, Genres et textes.

2. L'écrivain Daniel de Roulet l'a qualifié, pour cette raison, de pharaon et il a tourné ce raisonnement en dérision en ajoutant que si, "en Suisse, il est établi, depuis quelques années, que le nombre de chiens par habitant augmente et que le nombre d'enfants par couple diminue», il ne faut peut-être pas «en déduire que les femmes, en 
Suisse romande. Ce plaisant personnage a récidivé peu de temps après dans un journal de notre université - malheureusement sans subir la moindre contradiction : «Il y a une certaine corrélation, sur le plan international, entre le taux de maturités et le taux de chômage des détenteurs de maturité. ${ }^{3}$ Ce triste conseiller fédéral avait déjà révélé le fonds de commerce de sa politique lors du forum de l'EPFL, du Centre patronal vaudois et du journal 24 Heures, le 7 novembre 2012, en se disant "convaincu qu'une suracadémisation peut plus ou moins directement entraîner une désindustrialisation». Maintenant que la formation et la recherche sont intégrées dans son Département fédéral de l'économie, il est permis de redouter une "dé-science-humanisation» des hautes écoles, et je vous souhaite, chers collègues et chers étudiants, bien du courage.

En retraçant, comme le genre de la leçon d'adieu m'y autorise, mon parcours et les grandes orientations qui ont guidé mes travaux et mon enseignement lausannois, je mettrai l'accent sur le fait que la recherche est une pratique fondamentalement dialogique, un cheminement critique de savoir avec et contre les positions assumées par d'autres:

Une théorie dialogique [...] présuppose un Sujet théorique qui s'intéresse à la pensée de l'autre: à ses recherches, ses vérités et ses jugements de valeur. Elle exclut une indifférence postmoderne dans le cadre de laquelle toutes les positions, toutes les vérités apparaissent comme interchangeables. [...] Elle refuse un pluralisme indifférent qui concède à chaque individu son droit d'exprimer ses jugements de valeur pourvu qu'il reconnaisse la suprématie de la valeur d'échange et du marché ${ }^{4}$.

Ayant toujours trouvé inadmissible le gommage des dettes de pensée, je citerai très systématiquement celles et ceux qui m’ont fait avancer et qui m'aident encore à penser aujourd'hui ma place de linguiste du texte et du discours dans le champ des sciences de l'homme et de la société.

Suisse, ne font plus d'enfants mais des chiens» ("Johann Schneider-Ammann en pharaon", Le Temps, $1^{\mathrm{er}}$ novembre 2012).

3. F. Zambano, "Il faut renforcer les universités sans toucher à leur autonomie», entretien avec J. Schneider-Ammann, p. 17.

4. P. V. Zima, Théorie critique du discours, p. 16 sq. 


\section{Importance de Roman Jakobson et contexte de l'année 1970}

C'est en entendant, en février 1972, Roman Jakobson parler de poésie au Collège de France et du langage à la télévision française que j’ai eu envie de mettre mes pas dans ceux de cet immense savant. Quand je terminais mes études, en 1970, Jean Starobinski écrivait encore, dans sa préface aux Etudes de style de Leo Spitzer, que «[la] linguistique, science de la forme liée au sens, possède une application herméneutique dont l'intervention est la bienvenue partout où il y a du langage à lire et du sens à déchiffrer». Et il ajoutait: "La stylistique des chefs-d'œuvre n'est qu'une application - certes privilégiée - d'un savoir qui cesse de se confiner dans une neutralité prudente. ${ }^{5}$ En considérant les «limites spécialisées» de la linguistique comme le «reflet contingent d'une départementalisation académique ${ }^{6}$, il dessinait ce qui est devenu, pour moi, un programme de travail.

Il était enthousiasmant de finir ses études et de devenir chercheur l'année de la parution de Pour la poétique d'Henri Meschonnic, de la création de la revue Poétique, de la publication des Mots sous les mots par laquelle Starobinski nous rendait accessibles les cahiers de Saussure sur le vers saturnien bas latin et donnait la première transcription de la note manuscrite sur le discours dont j'ai mesuré, par la suite, l'importance. Par ailleurs, les travaux de Saussure sur les légendes germaniques ${ }^{7}$ annonçaient le Propp de la Morphologie du conte, traduit également en 1970. En décembre de cette année-là, Michel Foucault prononçait sa superbe leçon inaugurale sur "l'ordre du discours», au Collège de France (1971). Il situait l'analyse des "formations discursives» en deçà du niveau manifeste des constructions textuelles achevées:

Si elle étudie les modalités d'énonciation, [l'analyse des formations discursives] ne met en question ni le style ni l'enchaînement des phrases; bref, elle laisse en pointillé la mise en place finale du texte ${ }^{8}$.

Son objectif était clair:

5. J. Starobinski, «Leo Spitzer et la lecture stylistique», p. 10 et p. 38 sq.

6. Ibid., p. 10.

7. Cf. D’A. S. Avalle, «La sémiologie de la narrativité chez Saussure».

8. M. Foucault, L'archéologie du savoir, p. 100. 
Ce qui est analysé ici, ce ne sont certes point les états terminaux du discours, mais ce sont des systèmes qui rendent possibles les formes systématiques dernières; ce sont des régularités préterminales par rapport auxquelles l'état ultime, loin de constituer le lieu de naissance du système, se définit plutôt par ses variantes?

Se situant à un niveau "prédiscursif", Foucault faisait porter l'analyse de discours sur:

[...] un ensemble de règles anonymes, historiques, toujours déterminées dans le temps et l'espace qui ont défini à une époque donnée, et pour une aire sociale, économique, géographique ou linguistique donnée, les conditions d'exercice de la fonction énonciative ${ }^{10}$.

Il me paraissait nécessaire de développer, à côté de cette approche interdiscursive et de ce qui m’apparaissait comme un déficit de l'analyse de discours développée en France, une linguistique des agencements d'énoncés, jusqu'à leur rang le plus matériel de réalisation verbale. C'était l'orientation qu'allait prendre, au début des années 1980 seulement, le courant le plus dynamique de l'analyse de discours: celui qui se développait autour de Michel Pêcheux depuis son livre Analyse automatique du discours. Zellig S. Harris, dans les articles sur l'analyse du discours qui venaient d'être traduits en français en 1969, disait bien que:

La langue ne se présente pas en mots ou phrases indépendantes, mais en discours suivi, que ce soit un énoncé réduit à un mot ou un ouvrage de 10 volumes, un monologue ou un discours politique ${ }^{11}$.

Il ajoutait même cette idée sur laquelle j’ai beaucoup travaillé:

Le texte peut être constitué de morceaux successifs, sortes de soustextes à l'intérieur du texte principal, comme des paragraphes ou des chapitres ${ }^{12}$.

9. Ibid., p. 100 sq.

Io. Ibid., p. 153 sq.

II. Z. S. Harris, "Analyse du discours», p. 10 sq.

I2. Ibid., p. 24 sq. 
Malheureusement, la méthodologie mise en place par Harris restait trop limitée aux "réarrangements structurels au niveau de la phrase», comme l'ont déploré très tôt aussi bien Nicolas Ruwet que William Labov ${ }^{13}$.

A la différence des spécialistes anglo-saxons de grammaire de texte, j'ai beaucoup appris sur la textualité à la lumière des narratologies des années 1960-1970. La façon dont Barthes concevait la narratologie m'intéressait car, prolongeant explicitement l'article de Benveniste sur «Les niveaux de l'analyse linguistique " ${ }^{14}$, il s'efforçait de théoriser, sur le même principe structural, l'intégration des phrases dans un texte:

Si l'on essaie d'embrasser l'ensemble d'un récit écrit, on voit qu'il part du plus codé (le niveau phonématique, ou même mérismatique), se détend progressivement jusqu'à la phrase, point extrême de la liberté combinatoire, puis recommence à se tendre, en partant des petits groupes de phrases (micro-séquences), encore très libres, jusqu'aux grandes actions, qui forment un code fort et restreint: la créativité du récit $[. .$.$] se situerait ainsi entre deux codes, celui de la linguistique et$ celui de la translinguistique ${ }^{15}$.

Il développait ainsi une vision homologique de la phrase et du texte:

Le système du discours reproduit d'une façon en quelque sorte homographique le système de la phrase, avec ses deux coordonnées: d'une part, substitution, segmentation et relations distributionnelles entre segments d'un même niveau, et d'autre part, intégration des unités de chaque niveau dans une unité de niveau supérieur, qui lui donne sens ${ }^{16}$.

La description intégrative lui apparaissait comme une nécessité épistémologique et technique: «[...] c'est d'elle que dépend la segmentation du discours en unités." Barthes approchait ainsi la «limite du système»: «Le principe d'intégration [...] permet de donner un statut descriptif à la limite du système, en désignant, en termes sémiologiques (c'est là l'important), le moment où le système s'articule sur la praxis

I3. Voir N. Ruwet, «Parallélismes et déviations en poésie» et W. Labov, Le parler ordinaire, p. 223 sq.

I4. E. Benveniste, «Les niveaux de l'analyse linguistique».

I5. R. Barthes, "Introduction à l'analyse structurale des récits», p. 864.

I6. R. Barthes, "La linguistique du discours», p. 616. 
sociale et historique." Il considérait le genre comme le "premier niveau " d'intégration de la "suite hiérarchisée des autres niveaux" ${ }^{17}$.

Emile Benveniste ne posait pas le problème en termes de continuité, mais de saut de niveau. Affirmant que "le monde du signe est clos» et que «du signe à la phrase il n’y a pas transition, ni par syntagmation ni autrement», mais qu' "un hiatus les sépare», il en concluait que «[...] la langue comporte deux domaines distincts, dont chacun demande son propre appareil conceptuel » ${ }^{18}$. Faisant de la phrase "l'unité du discours», il la plaçait au centre d'un autre domaine linguistique: " [...] celui de la langue comme instrument de communication, dont l'expression est le discours " ${ }^{19}$. Dans "L'appareil formel de l'énonciation ", Benveniste semblait exclure - comme Foucault - le «texte de l'énoncé» du champ de la linguistique du discours et de l'énonciation:

Le discours, dira-t-on, qui est produit chaque fois qu'on parle, cette manifestation de l'énonciation, n'est-ce pas simplement la "parole»? - Il faut prendre garde à la condition spécifique de l'énonciation: c'est l'acte même de produire un énoncé et non le texte de l'énoncé qui est notre objet. Cet acte est le fait du locuteur qui mobilise la langue pour son compte ${ }^{20}$.

Mais il nuançait cette position en écrivant, à la fin de «Sémiologie de la langue", cette remarque programmatique que j'ai souvent commentée ${ }^{21}$ :

En conclusion, il faut dépasser la notion saussurienne du signe comme principe unique, dont dépendraient à la fois la structure et le fonctionnement de la langue. Ce dépassement se fera par deux voies:

- dans l'analyse intra-linguistique, par l'ouverture d'une nouvelle dimension de signifiance, celle du discours, que nous appelons sémantique, désormais distincte de celle qui est liée au signe, et qui sera sémiotique;

17. Ibid., p. 614, 616 et 615 .

I8. E. Benveniste, "Sémiologie de la langue», p. 65.

I9. E. Benveniste, «Les niveaux de l'analyse linguistique», p. 130.

20. E. Benveniste, «L'appareil formel de l'énonciation", p. 80.

2I. Cf. J.-M. Adam, «Les problèmes du discours poétique de Benveniste» et "Le programme de la "translinguistique des textes, des œuvres" et sa réception au seuil des années $1970 »$. 
- dans l'analyse translinguistique des textes, des œuvres, par l'élaboration d'une métasémantique qui se construira sur la sémantique de l'énonciation ${ }^{22}$.

Jusqu'à la publication, en 2011, par Chloé Laplantine, des notes de travail de Benveniste sur le "problème du discours poétique " ${ }^{23}$, nous ignorions qu'il était en train de développer cette "translinguistique des textes, des œuvres» et de préparer, pour le numéro 12 de Langages dirigé par Barthes et consacré à "Linguistique et littérature», un article sur la «langue de Baudelaire». Mais, engagé dans une réflexion plus ample et plus complexe, il n'a pas remis son article et, le 6 décembre 1969, un accident cérébral l'a réduit au silence. Pour moi, il fallait à tout prix poursuivre dans le sens qu'il indiquait en essayant de relever le défi posé par Volochinov:

La construction de la phrase complexe (la période), voilà tout ce que la linguistique peut prendre en compte. Quant à l'organisation de l'énoncé complet, elle en renvoie la compétence à d'autres disciplines: la rhétorique et la poétique. La linguistique n’a pas de méthode pour aborder les formes de composition d'un tout ${ }^{24}$.

Sous l'influence de mon premier professeur de linguistique, à l'Université de Haute-Normandie, Denis Slakta, je me suis tourné vers les travaux allemands de grammaire de texte ${ }^{25}$, les recherches du second Cercle de Prague, les premiers articles en français de Teun A. van Dijk ${ }^{26}$ et Tempus d'Harald Weinrich (dont la traduction française est parue en 1973).

En arrivant à Lausanne, en 1984, j'ai eu l'occasion de coordonner, avec Jean-Blaise Grize, Marie-Jeanne Borel et l'anthropologue Mondher Kilani, une recherche qui nous a collectivement menés, avec le remplacement de Grize par Claude Calame, à l'écriture d'un livre sur le

22. E. Benveniste, «Sémiologie de la langue», p. 66.

23. Cf. E. Benveniste, Baudelaire.

24. V. N. Volochinov, Marxisme et philosophie du langage, p. 281.

25. Voir D. Clément, B. Grünig (dir.), La grammaire générative en pays de langue allemande.

26. T. A. van Dijk, "Aspects d'une théorie générative du texte poétique» et «Modèles génératifs en théorie littéraire». 
discours anthropologique ${ }^{27}$. A l'occasion de cette recherche, la lecture de la théorie ethnographique du langage de Bronislaw Malinowski est venue confirmer mes choix théoriques. Cette lecture me renvoyait à la théorie anthropologique du langage de Wilhelm von Humboldt et à ces lignes de "Ueber die Verschiedenheiten des menschlichen Sprachbaues", que je cite dans la traduction d'Henri Meschonnic: "La langue consiste seulement dans le discours lié, grammaire et dictionnaire sont à peine comparables à son squelette mort. » ${ }^{28}$ De Humboldt, j'ai retenu que ce n'est que dans «les enchaînements du discours » que peuvent être perçus les éléments les plus significatifs de la langue. Bien avant la pragmatique et le "tournant actionnel» des sciences du langage, Malinowski soulignait que "[l]es mots participent de l'action et sont autant d'actions » ${ }^{29}$ et que leur puissance d'action ne peut être observée que dans le système des genres discursifs de la société trobriandaise du Pacifique Sud qu'il étudiait (formules magiques, exorcismes, malédictions, bénédictions, prières, ordres et les différentes formes de narration). Quinze ans avant l'émergence, au début du XX $\mathrm{X}^{\mathrm{e}}$ siècle, de l'analyse de discours ${ }^{30}$ et de la linguistique textuelle ${ }^{31}$, l'approche de Malinowski était très textuelle et discursive:

C'est aujourd'hui une vérité première de la linguistique que la plus petite unité de la langue n'est pas le mot, mais la phrase. Ce qu'il s'agit de montrer, c'est que même la phrase n'est pas une unité autonome du discours, qu'elle ne se suffit pas à elle-même. [...] Une phrase apparaît généralement au milieu d'autres phrases, et n'a de sens que si on la considère comme un élément d'une totalité significative qui la dépasse. Je crois qu'on a tout intérêt, en linguistique, à élargir la notion de contexte $[\ldots]^{32}$.

Tout cela rappelle un célèbre aphorisme de Schleiermacher: "Toute compréhension du détail est conditionnée par une compréhension

27. J.-M. Adam et al. (éds), Le discours anthropologique.

28. H. Meschonnic, Langage, histoire, p. 630.

29. B. Malinowski, Les jardins de corail, p. 243.

30. Z. S. Harris, "Analyse du discours".

31. E. Coseriu, «Determinación y entorno».

32. B. Malinowski, Les jardins de corail, p. 257. 
du tout» ${ }^{33}$, dont Volochinov a tiré toutes les conséquences pour la linguistique:

Tant que l'énoncé considéré comme un tout restera terra incognita pour le linguiste, il ne saura être question de comprendre de façon réelle, concrète, non scolastique, une forme syntaxique.

Nous avons déjà dit qu'en linguistique tout ne va pas pour le mieux dès qu'il s'agit de la totalité formée par l'énoncé. On peut dire carrément que la réflexion linguistique a irrémédiablement perdu le sentiment de la parole envisagée comme un tout. C'est au milieu d'une phrase que le linguiste se sent le plus à l'aise. Plus il se rapproche des confins de la parole, de l'énoncé en tant que tout, moins sa position est sûre. Il n'a aucun moyen pour aborder la totalité; aucune des catégories linguistiques ne convient pour définir une totalité ${ }^{34}$.

Volochinov concluait de façon très proche de Benveniste:

C'est pourquoi il n'y a ni transition progressive, ni même aucun lien entre les formes linguistiques des éléments de l'énoncé et celles de la totalité qu'il constitue. Ce n'est qu'en faisant un saut qualitatif qu'on passe de la syntaxe aux questions de composition. Cela est inévitable, puisque l'on ne peut percevoir et comprendre les formes d'un énoncé en tant que totalité que sur le fond des autres énoncés formant eux-mêmes une totalité dans l'unité d'une même sphère idéologique donnée ${ }^{35}$.

Mais, pour théoriser ce saut qualitatif, également posé par Halliday et Hasan $^{36}$, il fallait entrer dans une épistémologie de la complexité dont Jakobson, dans son discours de clôture du congrès de 1952, avait dessiné les grandes lignes, en disant à propos de la nécessaire spécialisation et de l'étude de problèmes limités:

Nous savons qu'il s'agit là seulement de différents modes d'expérimentation, non de points de vue exclusifs. [...] Nous ne pouvons pas vraiment isoler les éléments, mais seulement les distinguer. Si nous sommes amenés à les traiter séparément au cours du processus de

33. F. D. E. Schleiermacher, Herméneutique, p. 67.

34. V. N. Volochinov, Marxisme et philosophie du langage, p. 353.

35. Ibid., p. 281.

36. M. A. K. Halliday, R. Hasan, Cohesion in English, p. 293. 
l'analyse linguistique, nous devons toujours nous souvenir du caractère artificiel d'une telle séparation ${ }^{37}$.

La différence entre «distinguer» et «isoler» est fondamentale: on «isole» dans une épistémologie réductionniste et on "distingue» dans une théorie de la complexité. Dans une "théorie de la complexité", des sauts qualitatifs doivent être pensés comme l'émergence de faits non prévisibles par simple somme de leurs composants ${ }^{38}$. Le sens d'une phrase est bien plus que la somme des signes qui la composent; un texte est une unité bien plus complexe que la simple somme des phrases qui constituent ses paragraphes; un discours est bien plus que la somme des niveaux de textualisation qui font du texte une unité de sens en contexte.

\section{Interdisciplinarité critique et paradigmes du texte}

Comme je le disais plus haut, j'ai beaucoup travaillé sur les narratologies de l'époque dont, bien sûr, la sémiotique narrative et la poétique, mais aussi les approches psycho-cognitives du récit ${ }^{39}$. J'ai également travaillé sur la poétique de la description de Philippe Hamon ${ }^{40}$ et sur les théories de l'argumentation développées à l'Université Libre de Bruxelles autour de Chaïm Perelman et dans le cadre du Centre de recherches sémiologiques de Neuchâtel, autour de Jean-Blaise Grize et de Marie-Jeanne Borel. Bien que très différents, ces domaines avancés de la recherche m'aidaient à penser la linguistique textuelle autrement que comme une grammaire et une typologie de textes. Je dois dire aussi la stimulation du dialogue que j'ai pu mener, en Suisse, avec l'ambitieux modèle modulaire d'Eddy Roulet, extension des propositions théoriques du Danois Henning Nølke, et surtout avec l'interactionnisme socio-discursif développé à Genève par Jean-Paul Bronckart ${ }^{41}$.

37. R. Jakobson, Essais de linguistique générale, p. 26.

38. Cf. J.-M. Adam, "Le "bougé du texte" dans la théorie du langage».

39. Cf. M. Fayol, Le récit et sa construction.

40. Ph. Hamon, Du descriptif.

4I. Cf. E. Roulet, "Une approche modulaire de la complexité de l'organisation du discours", H. Nølke, "Linguistique modulaire» et J.-P. Bronckart, Activité langagière, textes et discours. 
Je tenais de l'enseignement de Denis Slakta une mise en garde que je n’ai jamais oubliée: «La "pureté" de la linguistique est une illusion", disait-il, et il déplorait que soit «rejeté dans l'enfer de l'extra-linguistique, non seulement ce qui est notion idéologique [...] mais aussi tout ce qui a rapport à d'autres sciences». Tout en se méfiant d'une «vague pluridisciplinarité où l'ignorance d'un seul serait multipliée par les ignorances de tous", il affirmait la nécessité "d’articuler la science linguistique à d'autres sciences si l'on veut sortir de l'impasse où certains entendent confiner les linguistes " ${ }^{42}$. Mon projet a toujours consisté moins à chercher à «articuler" qu' à penser le continu des problèmes qu'abordent et dévoilent, de façon discontinue, les sciences des textes; moins à «articuler» qu’à penser ce que Meschonnic appelait, dans Pour la poétique, «une pratique du continu vers une pensée du continu» ${ }^{43}$.

C'était tout le sens du séminaire interdisciplinaire lausannois qui a réuni pendant des années des philosophes, des littéraires, des antiquisants et des linguistes, et même des géographes et des historiens, sous la houlette de Marie-Jeanne Borel et de Claude Calame ${ }^{44}$. Après la retraite prématurée de la première, Ute Heidmann a rapidement joué un rôle comparable de mise en relation des disciplines et de mise en ouvre de l'interdisciplinarité impliquant d'abord des comparatistes et des antiquisants, mais aussi des anglicistes et des italianistes - dont notre regretté collègue Marco Praloran, auquel je dédie cette leçon -, des littéraires et des linguistes. Sous l'impulsion du rectorat de l'Université, nous avons, Ute Heidmann et moi, contribué à la création de ce qui est devenu la Formation doctorale interdisciplinaire de la Faculté des lettres, confiée par la suite à Jérôme Meizoz, et deux centres de recherche et d'enseignement à vocation interdisciplinaires (le Centre de recherches en langues et littératures européennes comparées et le Centre des sciences historiques de la culture). Dans notre esprit, il s'agissait de développer avec une grande exigence, la dynamique que résume Meschonnic dans son dernier livre:

42. D. Slakta, «Esquisse d'une théorie lexico-sémantique», p. 93.

43. H. Meschonnic, Pour la poétique, p. 7.

44. J'ai résumé l'histoire de ce séminaire dans la postface de Poétiques comparées des mythes, volume d'hommage à Claude Calame mis au point et coordonné par Ute Heidmann (Payot, 2003). 
Il faut une critique de la linguistique par la philosophie et de la philosophie par la linguistique, de la philologie par la poétique et de la poétique par la philologie, de la sociologie par la poétique et de la poétique par la sociologie, et ainsi de proche en proche la critique de chacune des sciences humaines et de toutes ensemble par l'examen du statut qu'elles réservent au langage: leur sens du sens ${ }^{45}$.

Notre projet était très différent de la confortable juxtaposition pluridisciplinaire que certains pratiquent pour éviter soigneusement tout retour critique sur leurs propres positions et l'«ébranlement» des certitudes qu'un dialogue interdisciplinaire peut entraîner. Comme le dit très bien Peter V. Zima, ce «dialogue théorique» entre des positions hétérogènes n'est possible qu'à condition de rendre nos positions et présupposés disciplinaires explicites, de reconnaître la nature discursive de nos constructions scientifiques ainsi que l'historicité de leurs conditions de constitution, et surtout à condition d'accepter l'effort de décentrement disciplinaire que cela implique ${ }^{46}$.

Le programme interdisciplinaire des Essais de linguistique générale de Jakobson ${ }^{47}$ relevait de cette interdisciplinarité critique. Les espoirs de Jakobson étaient immenses quand il pensait, en 1952, que «les problèmes du langage poétique [étaient] désormais au premier plan des préoccupations des linguistes américains» et qu’un pont était enfin jeté "entre la linguistique et la critique littéraire de ce pays " ${ }^{48}$. Sa célèbre conclusion du grand colloque interdisciplinaire sur le style allait dans le même sens. Malheureusement, comme de nombreuses publications récentes le prouvent et pour reprendre les mots de Jakobson, les «linguistes sourds à la fonction poétique» se sont multipliés, comme les

45. H. Meschonnic, Langage, histoire, p. 630.

46. Cf. P. V. Zima, Théorie critique du discours, p. I6.

47. R. Jakobson n'est qu'un des acteurs de l'incroyable densité de réflexion sur le langage et l'art verbal littéraire développée en Russie (puis Union soviétique) et à Prague pendant le premier tiers du XXe siècle. La caractéristique majeure de ce bouillonnement de la pensée incarné par Jakobson est l'apport conjoint de toutes les sciences de l'homme et de la société; de l'art verbal à la psychologie sociale, en passant par l'ethnoanthropologie, il faudrait associer les noms de Vygotski, Volochinov, Jakubinski, Bogatyrev, Tynianov, Troubetzkoy à ceux d'écrivains et de peintres futuristes russes.

48. R. Jakobson, Essais de linguistique générale, p. 30. 
«spécialistes de la littérature indifférents aux problèmes et ignorants des méthodes linguistiques» ${ }^{49}$.

C'est contre ces «flagrants anachronismes» pointés par Jakobson que j'ai travaillé dès mon premier livre, écrit avec la collaboration de JeanPierre Goldenstein (1976), et cela jusqu'au travail mené ces dix dernières années avec Ute Heidmann, comparatiste spécialiste du champ littéraire, sur les contes de Perrault, d'Andersen et des Grimm, des textes de Kafka et l'intertextualité faustienne des "Chats» de Baudelaire. Ces années de recherche et de confrontation des problématiques littéraire, comparatiste et linguistique ont donné deux livres qui montrent l'utilité de penser les problèmes ensemble et que j'aurais été incapable d'écrire seul: Le texte littéraire. Pour une approche interdisciplinaire (2009) et Textualité et intertextualité des contes (2010). Profitant par ailleurs du travail scientifique mené par Ute Heidmann avec l'épistémologue Silvana Borutti et avec Henri Meschonnic, j'ai mesuré l'importance des paradigmes de la traduction et de la comparaison. J'ai aussi compris ce que dit Jean-Marie Schaeffer dans Petite écologie des études littéraires:

Concevoir, par exemple, la littérature anglaise comme un tout (holos) revient à l'isoler des autres, donc à rendre, de proche en proche, toutes les littératures incommensurables entre elles. Le fait que, de nos jours encore, la littérature comparée reste une discipline marginale [...] (alors qu'elle devrait être la reine des disciplines littéraires, si celles-ci s'intéressaient réellement aux faits littéraires dans leur diversité et leur unité) est un signe parlant de cette incapacité des études littéraires à faire la différence entre leur objet d'étude - les faits littéraires - et les diverses constructions nationales de "La Littérature» 50 .

Gilles Philippe et Dominique Maingueneau ont également insisté sur les vertus heuristiques et méthodologiques de la comparaison:

Nous privilégions, en outre, les analyses comparatives, démarche étrangère aux commentaires stylistiques et aux explications de textes traditionnels, qui abordent les extraits comme des totalités autosuffisantes. Il nous semble, en effet, que la confrontation est éclairante: des œuvres qui paraissent très différentes peuvent se révéler proches, d'autres qui se réclament de la même esthétique peuvent diverger

49. Voir ibid., p. 248.

50. J.-M. Schaeffer, Petite écologie des études littéraires, p. 22. 
considérablement. De manière plus large, la comparaison permet d'attirer l'attention sur des phénomènes qui, sans cela, auraient été négligés ${ }^{51}$.

Dans cet esprit, l'intérêt des propositions d'Ute Heidmann ${ }^{52}$ réside dans la théorisation de la "comparaison différentielle» dont elle applique les principes au traduire, aux réécritures des mythes et des contes et à d'autres genres. L'avantage de cette "analyse comparée des discours" est de mettre l'accent sur les "différences" et, plus précisément, sur les "différenciations» des langues, des littératures et des cultures. En considérant le texte d'origine et sa traduction comme deux énonciations singulières dont chacune construit ses effets de sens en se liant de façon significative à son propre contexte socioculturel et linguistique, Ute Heidmann montre que le rapport entre un texte original et ses traductions fonctionne sur le mode du "dialogue intertextuel", intergénérique et interculturel. La comparaison différentielle met en évidence les modalités d'un dialogue entre textes et genres qui est partie intégrante du "principe dialogique» ${ }^{53}$. Dans le cadre du Centre de recherche en langues et littératures européennes comparées, je me suis ainsi de plus en plus intéressé à l'approche textuelle, comparative et discursive de la traduction et à la place de l'interprétation dans les sciences et disciplines des textes ${ }^{54}$.

Au début des années 1970, prenant acte du fait que les sciences de l'homme et de la société ont, à la différence des sciences de la nature, le symbolique pour objet, Paul Ricœur avait fait du «paradigme de l'interprétation textuelle» un fondement de la méthode des sciences humaines et sociales. Son «herméneutique critique» déconstruisait, à la fois, la

5I. D. Maingueneau, G. Philippe, Exercices de linguistique pour le texte littéraire, p. V.

52. Voir U. Heidmann, "Comparatisme et analyse de discours», "Epistémologie et pratique de la comparaison différentielle», "Enjeux d'une comparaison différentielle et discursive», "La comparaison différentielle comme approche littéraire».

53. Cf. T. Todorov, Mikhaïl Bakhtine, le principe dialogique.

54. Les 4 et 5 octobre 2007, le colloque de fondation du Centre de recherche en langues et littératures européennes comparées organisé par Ute Heidmann réunissait exemplairement des chercheurs suisses, français, italiens, allemands et belges comme Henri Meschonnic, Silvana Borutti, François Ost, Heinz Wismann, Sophie Klimis, Emmanuelle Danblon. 
tradition romantique en herméneutique et le modèle d'explication issu des sciences naturelles et de leur logique inductive. Ricœur fondait sa démarche sur une définition du texte comme "paradigme de la distanciation dans la communication", révélateur «de l'historicité même de l'expérience humaine, à savoir qu'elle est une communication dans et par la distance " ${ }^{55}$. Cette idée vient de l'herméneutique de Hans Georg Gadamer, qui utilise le concept de «distance» pour critiquer la conception romantique de la "compréhension». Dans leur dernier livre, Silvana Borutti et Ute Heidmann rappellent que la distance est un élément constitutif de la possibilité de la compréhension d'autrui et elles ajoutent:

Face à un texte et à une œuvre, la question que se pose l'approche interprétative est: comment reconstruire un système symbolique étranger à partir de son étrangeté? La distance est envisagée alors comme la structure fondamentale du comprendre, c'est-à-dire de l'accès au sens de l'autre. C'est un thème cognitif et en même temps ontologique, qui est au cœur de la question du "comprendre» dans les sciences humaines et dans les études interculturelles ${ }^{56}$.

Elles insistent sur le fait que la distance, accentuée par «l'état de suspension entre deux langues et deux cultures", rend possible "l'accès au sens de l'autre " ${ }^{57}$. La traduction permet de "penser entre les langues", selon la formule en titre du dernier livre d'Heinz Wismann, et donc «dans le langage», mais elle présente un autre avantage: celui de penser, par la comparaison, «entre les textes». Comme l'a dit et répété Meschonnic: «La traduction est le mode le plus banal, le plus admis, le plus visible des transformations qui font qu'un texte est à la fois toujours le même et un autre.» Et il ajoute: «Une traduction n'est [...] qu'un moment d'un texte en mouvement. Elle est même l'image qu'il n'est jamais fini.» ${ }^{58}$ Cette idée est au cœur de l'œuvre de Jorge Luis Borges:

Qu'est-ce qu'un livre si nous ne l'ouvrons pas? Un simple cube de papier et de cuir, avec des feuilles; mais si nous le lisons, il se passe quelque chose d'étrange, je crois qu'il change à chaque fois.

55. P. Ricœur, Du texte à l'action, p. 102.

56. S. Borutti, U. Heidmann, La Babele in cui viviamo, p. 85; je traduis.

57. Ibid., p. 84 et 85 ; je traduis.

58. H. Meschonnic, Poétique du traduire, p. 175 et 342. 
Héraclite dit (je l'ai trop souvent répété) qu'on ne se baigne jamais deux fois dans le même fleuve. On ne se baigne jamais deux fois dans le même livre parce que ses eaux changent mais le plus terrible est que nous ne sommes pas moins fluides que le fleuve. Chaque fois que nous lisons un livre, le livre a changé, la connotation des mots est autre. En outre, les livres sont chargés de passé 59 .

Je ne prendrai, faute de place, comme exemple que le titre du recueil intitulé El Hacedor, dont Borges dit, dans le texte-épilogue du recueil:

De tous les livres que j'ai confiés à l'impression, aucun, je crois, n'est aussi personnel que cette forêt de leçons variées arbitrairement assemblées et désordonnées, précisément parce qu'elle abonde en reflets et en interpolations ${ }^{60}$.

Si on prend au sérieux cette idée d' "interpolation", on doit être sensible aux reflets et aux échos entre textes du recueil, même les plus éloignés et génériquement différents. C’est une instruction de lecture et de traduction.

En langue, le syntagme nominal El Hacedor est dérivé du verbe espagnol hacer («faire», en français). Roger Caillois, traducteur et introducteur de Borges en France, a bien perçu la présence d'une ressemblance avec le grec ancien, mais il prend, dans l'«Avertissement» de sa traduction, une décision qui ne me semble pas heureuse:

Le titre du recueil El Hacedor est tiré du verbe «faire» (hacer), comme le grec poietes (poète) du verbe correspondant (poïein, faire). La traduction correcte aurait donc été faiseur, si le mot n'avait pas pris en français divers sens dont aucun ne convient en l'occurrence. Fabricant, fabricateur, artisan, ouvrier n'étaient pas moins inopportuns. Je me suis donc résigné à traduire par auteur ${ }^{61}$.

Les traductions anglaise (The Maker), italienne (L'Artefice), roumaine (Făuritorul) ou brésilienne (O Fazedor) rendent plus sensible le fait que le concept d' "Auteur» efface les connotations artisanales du verbe «faire» conservées dans ces autres langues et rappelées dans une conférence donnée par Borges à Harvard, en 1967: «Je crois qu’un jour le poète sera de

59. J. L. Borges, Conférences, p. 157.

6o. J. L. Borges, L'auteur et autres textes, p. 214 ; je traduis.

6I. Ibid., p. 9 sq. 
nouveau le créateur, le faiseur au sens antique. " ${ }^{62}$ Caillois est manifestement gêné par l'interlangue gréco-hispanique, comparable au MakerPoietes de l'anglo-saxon ancien. Il néglige, de ce fait, la saturation de la langue du recueil par un grand nombre d'«interpolations» et de «reflets" culturels antiques. Ainsi dans le premier texte du recueil, qui parle d'un homme en train de devenir aveugle - comme Borges:

En cette nuit de ses yeux mortels où il descendait maintenant, l'attendaient aussi bien l'amour que le danger. Arès et Aphrodite, parce qu'il devinait déjà (parce que déjà l'entourait) un bruissement de gloire et d'hexamètres, un bruissement d'hommes qui défendent un temple que les dieux ne sauveront pas et de vaisseaux noirs qui cherchent par les mers une île aimée, un bruissement d'Odyssées et d'Iliades que son destin était de chanter et de laisser résonner concavement dans la mémoire humaine ${ }^{63}$.

La traduction française oublie le "primat de l'organisation textuelle sur les unités» ${ }^{64}$. Borutti et Heidmann le disent en se référant à la critique, par W. van O. Quine, de l'atomisme et du dogme réductionniste:

Dire qu'on ne passe pas d'une langue à une autre, d'un terme à un autre, mais d'un énoncé à un autre, c'est faire allusion au fait qu'en traduisant nous faisons l'hypothèse de correspondances entre des occurrences textuelles [...]. Disant que nous traduisons d'énoncé à énoncé, Quine se réfère au fait que, dans la traduction, on doit tenir compte non seulement de la dépendance du sens à l'égard de l'ensemble immanent et autoréférentiel des signes de la langue, mais aussi de leur intégration dans l'ensemble du texte ${ }^{65}$.

Dans le Magazine littéraire du mois de juin 2012, Alberto Manguel, qui a bien connu Borges, parle du côté «artisan, horloger, mécanicien" de l'écrivain argentin, très attentif à la construction de ses textes et à la grammaire de la langue:

Quand Borges appelle El Hacedor son livre qui en français s'intitule L'Auteur et autres textes, hacedor est la traduction littérale de maker, du

62. J. L. Borges, L'art de poésie, p. 55.

63. J. L. Borges, L'auteur et autres textes, p. 20 ; je traduis.

64. H. Meschonnic, Poétique du traduire, p. 335.

65. S. Borutti, U. Heidmann, La Babele in cui viviamo, p. 58 sq.; je traduis. 
mot pour "poète» dans l'anglo-saxon ancien, mais dans le sens d'artisan. La traduction aurait dû être non pas L'Auteur, mais "L'Artisan", celui qui construit quelque chose avec ses mains, très concrètement ${ }^{66}$.

Pour traduire El Hacedor, j'hésite donc entre «L'Artisan», comme le propose Manguel, et "Le poëte", graphié à la manière de Baudelaire et de Mallarmé, avec un tréma sur le /e/, afin d'introduire une distance, de signaler la présence de l'interlangue pö̈etes-hacedor que construit intertextuellement et interculturellement le recueil. Cette traduction rendrait surtout génériquement visibles les poèmes sous les textes en prose et elle relancerait l'attention lectoriale et traductoriale. Ce que n'a pas fait Caillois, comme je le montre plus en détail dans le dernier chapitre de La linguistique textuelle, à propos d'un texte de ce recueil ${ }^{67}$.

Traduire (et plus largement lire-interpréter) ne consiste pas seulement à faire passer des énoncés d'une langue (l'espagnol d'Argentine) dans une autre (le français littéraire standard), mais à passer, et c'est beaucoup plus complexe, par une troisième langue: celle d'un livre, d'un recueil et même de chacun de ses textes. D'un état génétique à un autre, d'une édition à une autre, comme d'une traduction à une autre, c'est le texte lui-même qui se transforme, et les conséquences sur la théorie du texte sont importantes, car ce qui devient signifiant, ce qui fait sens, c'est le «bougé du texte» dont parle Meschonnic:

Ce qui bouge dans un texte, et par lui, c'est les notions de langage qu'il met en ouvre, qui se modifient selon la reconnaissance du texte, autant que le texte se modifie selon la transformation des notions avec lesquelles on le lit. A travers le bougé d'un texte, c'est la notion de texte elle-même qui bouge ${ }^{68}$.

Nous nous éloignons ainsi de la traductologie, mais aussi du textualisme structuraliste, avec sa "clôture du texte», et des dérives post-textualistes contemporaines. La distanciation que j'oppose à la "lecture actualisante" est une expérience de l'altérité et de l'étrangeté de toutes les productions symboliques. C'est ainsi que j'ai toujours compris l'herméneutique de la

66. A. Gefen, «Le monde comme livre et le sacre du lecteur», entretien avec Alberto Manguel, p. 54.

67. J.-M. Adam, La linguistique textuelle, p. 269-287.

68. H. Meschonnic, Poétique du traduire, p. 174. 
différence de Jean Starobinski ${ }^{69}$, qui m'apparaît comme le contraire d'une herméneutique de l'appropriation actualisante: «La différence reconnue est la condition de toute rencontre authentique." A quoi Starobinski ajoute cette mise en garde: "En sauvegardant la conscience de sa différence - condition de sa relation - le critique écarte le risque du monologue. ${ }^{70}$

C'est à partir de cette expérience de la "distance» et de la «différence» que peuvent surgir des effets de connaissance et des effets de formation intellectuelle: la difficulté acceptée de la langue d'un texte, toujours à traduire, d'une certaine manière - intra- et interlinguistique, voire intersémiotique - est une épreuve du sens pour un sujet confronté à un objet complexe. Cette épreuve est formatrice pour l'esprit, et l'université est le seul lieu où les jeunes chercheurs et futurs enseignants peuvent y être initiés. Il faut du temps et des savoirs méthodologiques pour mettre en place le décentrement de soi qui seul permet d'affronter les résistances qui activent et relancent le processus de compréhension et qui rendent possible l'invention et la créativité critiques, ce que Starobinski appelle la "disponibilité réflexive». L'enseignement, à tous ses niveaux, doit être dynamisé par l'effort d'adaptation et de décentrement critique que la confrontation aux textes émanant d'autres époques, langues et cultures, et plus largement aux productions de l'art verbal, rend possible. C'est le but de la comparaison entre un texte et ses traductions, un texte et ses états génétiques ou éditoriaux, un texte et ses intertextes ou des textes génériquement apparentés.

\section{Pour conclure}

La question préalable à tout dialogue entre sciences et disciplines du texte est celle de l'état épistémologique des théories. J'ai parlé ailleurs des conditions épistémologiques qui rendent possible un dialogue de la linguistique du texte et du discours avec ce qu'on appelle aujourd'hui la "génétique», mais aussi avec l'«histoire du livre et de l'édition" et avec la "philologie ${ }^{71}$, du moins, quand elle prend la forme d'une

69. Cf. J. Starobinski, La relation critique.

70. J. Starobinski, «Leo Spitzer et la lecture stylistique», p. 52 et 50.

7I. Voir J.-M. Adam, «Récriture et variation» et «Les sciences de l'établissement du texte et la question de la variation ". 
philologie textuelle et critique, fondée sur une herméneutique critique et sur le refus radical de toute position théologique ${ }^{72}$. C'était tout l'enjeu des travaux menés, avec Ute Heidmann, sur les contes de Perrault, des Grimm et d'Andersen. Pour rendre les contes à leur historicité critique d'événements de discours, il fallait commencer par les lire de très près en faisant tout le contraire du "distant reading" de Franco Moretti ${ }^{73}$. Au lieu de lire les textes, ce «lire de loin " consiste à cartographier et à quantifier la production romanesque mondiale sur la base d'informations de seconde main prises pour des «faits» (data). Comment peut-on prendre ce retour à un positivisme des années 1960 pour l'avenir des humanités littéraires et du comparatisme? Ute Heidmann s'en étonnait déjà dans "Comparatisme et analyse de discours", où elle insistait sur la conséquence la plus sensible de cette position: la perte de notre objet avec l'exclusion des textes et des langues ${ }^{74}$.

J'ai toujours pensé que c'est à élever les capacités de "lire de très près » que je devais former de futurs citoyens, dans l'espoir de les rendre moins naïfs, de les aider à se forger des instruments critiques à la fois solides et transférables dans d'autres domaines de leurs études et de leur vie sociale. Le cadre épistémologique et méthodologique dont j’ai parlé tout au long de cette "dernière séance" m'a permis de suivre, dans ma recherche et dans mes enseignements, le programme fixé en 1975 et confirmé en 1978 par Tzvetan Todorov, qui insistait sur la nécessité de prendre en compte les genres de discours en déconstruisant les séparations disciplinaires et en repensant le champ même des humanités:

A la place de la seule littérature apparaissent maintenant de nombreux types de discours qui méritent au même titre notre attention. [...] Un champ d'études cohérent, pour l'instant découpé impitoyablement entre sémanticiens et littéraires, socio- et ethnolinguistes, philosophes du langage et psychologues, demande impérieusement à être reconnu, où la poétique cédera sa place à la théorie du discours et à l'analyse de ses genres ${ }^{75}$.

Jean-Michel AdAm

72. Cf. F. Rastier, Arts et sciences du texte.

73. Voir F. Moretti, La letteratura vista da lontano et Graphes, cartes et arbres.

74. Voir U. Heidmann, "Comparatisme et analyse de discours", p. 100 sq.

75. T. Todorov, Les genres de discours, p. 26. 


\section{BIBLIOGRAPHIE}

Adam, Jean-Michel, Eléments de linguistique textuelle, Bruxelles, Mardaga, 1990.

—, "Barthes en 1970: de la translinguistique à la déconstruction", in Littérature et sciences humaines, éd. par Alain Boissinot et al., Paris, Les Belles-Lettres / CRTH de l'Université de CergyPontoise, 2001, p. 125-148.

—, "Postface», in Poétiques comparées des mythes. De l'Antiquité à nos jours, éd. par Ute Heidmann, Lausanne, Payot, 2003, p. 243-256.

-, "Les sciences de l'établissement du texte et la question de la variation", in Sciences du texte et analyse de discours, éd. par JeanMichel Adam, Ute Heidmann, Genève, Slatkine, 2005, p. 69-92.

—, "Récriture et variation: pour une génétique linguistique et textuelle", Modèles linguistiques, XXX (2009), vol. 59, p. 21-31.

—, La linguistique textuelle. Introduction à l'analyse textuelle des discours, Paris, Armand Colin, 2011 (2005).

-, Genres de récits. Narrativité et généricité des textes, Louvain-la-Neuve, Academia L'Harmattan, 2011.

—, Les textes: types et prototypes, Paris, Armand Colin, 2011 (1992).

—, "Le programme de la "translinguistique des textes, des œuvres" et sa réception au seuil des années 1970 ", in Relire Benveniste, éd. par E. Brunet, R. Mahrer, Louvain-la-Neuve, Academia, 2011, p. 123-147.

—, "Les problèmes du discours poétique de Benveniste. Un parcours de lecture», Semen, 33 (2012), p. 25-54.

—, «Le modèle émergentiste en linguistique textuelle», L'information grammaticale, 134 (2012), p. 30-37.

—, "Le "bougé du texte" dans la théorie du langage. Meschonnic et Borges", Europe, 995 (2012), p. 186-193.

Adam, Jean-Michel, Borel, Marie-Jeanne, Calame, Claude, Kilani, Mondher (éds), Le discours anthropologique, Paris, Méridiens Klincksieck, 1990. 
Adam, Jean-Michel, Goldenstein, Jean-Pierre, Linguistique et discours littéraire, Paris, Larousse, 1976.

Adam, Jean-Michel, Heidmann, Ute (éds), Sciences du texte et analyse de discours, Genève, Slatkine, 2005.

Adam, Jean-Michel, Heidmann, Ute, Le texte littéraire. Pour une approche interdisciplinaire, Louvain-la-Neuve, Academia Bruylant, 2009.

Adam, Jean-Michel, Laplantine, Chloé (dir.), Les notes manuscrites de Benveniste sur la langue de Baudelaire, Semen, 33 (2012).

Avalle, D’Arco Silvio, "La sémiologie de la narrativité chez Saussure», in Essais de la théorie du texte, éd. par Charles Bouazis et al., Paris, Galilée, 1973, p. 17-49.

BARthes, Roland, "Introduction à l'analyse structurale des récits" (1966), in Euvres complètes, Paris, Seuil, 2002, vol. 2, p. 828-865.

—, "La linguistique du discours" (1970), in Euvres complètes, Paris, Seuil, 2002, vol. 3, p. 611-616.

Benveniste, Emile, "Les niveaux de l'analyse linguistique", in Problèmes de linguistique générale, Paris, Gallimard, 1966, vol. 1, p. 119-131.

—, "Sémiologie de la langue», in Problèmes de linguistique générale, Paris, Gallimard, 1974, vol. 2, p. 43-66.

—, "L'appareil formel de l'énonciation", in Problèmes de linguistique générale, Paris, Gallimard, 1974, vol. 2, p. 79-88.

—, Baudelaire, éd. par Chloé Laplantine, Limoges, Lambert-Lucas, 2011.

Borges, Jorge Luis, L'auteur et autres textes, traduit par Roger Caillois, édition bilingue, Paris, Gallimard, 1982 (1960).

—, Conférences, Paris, Gallimard, 1985 (1980).

—, L'art de poésie, édition bilingue, Paris, Gallimard, 2002 (2000).

Borutti, Silvana, Heidmann, Ute, La Babele in cui viviamo, Torino, Boringhieri, 2012.

Bronckart, Jean-Paul, Activité langagière, textes et discours. Pour un interactionnisme socio-discursif, Paris/Lausanne, Delachaux et Niestlé, 1997.

Clément, Danièle, Grünig, Blanche (dir.), La grammaire générative en pays de langue allemande, Langages, 26 (1972). 
Coseriu, Eugenio, «Determinación y entorno. Dos problemas de una lingüística del hablar», Romanistisches Jahrbuch, 7 (1955-1956), p. 29-54.

Dijk, Teun Adrianus van, "Aspects d'une théorie générative du texte poétique", in Essais de sémiotique poétique, éd. par A.J. Greimas et al., Paris, Larousse, 1972, p. 180-206.

—, "Modèles génératifs en théorie littéraire», in Essais de la théorie du texte, éd. par Charles Bouazis et al., Paris, Galilée, 1973, p. 79-99.

—, "Grammaires textuelles et structures narratives", in Sémiotique narrative et textuelle, ouvrage présenté par Claude Chabrol, Paris, Larousse, 1973, p. 177-207.

FAYOL, Michel, Le récit et sa construction, Paris/Lausanne, Delachaux et Niestlé, 1985.

Foucault, Michel, L’archéologie du savoir, Paris, Gallimard, 1969.

—, L'ordre du discours, Paris, Gallimard, 1971.

Gefen, Alexandre, "Le monde comme livre et le sacre du lecteur", entretien avec Alberto Manguel, Magazine Littéraire, 520 (2012), p. 54-55.

Halliday, Michael Alexander Kirkwood, Hasan, Ruqaiya, Cohesion in English, London, Longman, 1976.

Hamon, Philippe, Du descriptif, Paris, Hachette, 1993 (1981).

Harris, Zellig Sabbetai, "Analyse du discours" (1952), Langages, 13 (1969), p. 8-45.

Heidmann, Ute (éd.), Poétiques comparées des mythes. De l'Antiquité à nos jours, Lausanne, Payot, 2003.

—, "Comparatisme et analyse de discours. La comparaison différentielle comme méthode", in Sciences du texte et analyse de discours, éd. par Jean-Michel Adam, Ute Heidmann, Genève, Slatkine, 2005, p. 99-118.

—, «Epistémologie et pratique de la comparaison différentielle. L'exemple des (ré)écritures du mythe de Médée", in Comparer les comparatismes, éd. par Maya Burger, Claude Calame, Paris/ Milan, Edidit/Arché, 2006, p. 141-159.

—, "Enjeux d'une comparaison différentielle et discursive. L'exemple de l'analyse des contes", in Les nouvelles voies du comparatisme, éd. par Hubert Roland, Stéphanie Vanasten, Gent, Ginko Academia Press, 2010, p. 27-40 (Cahiers voor literaturwetenschap 2). 
—, "La comparaison différentielle comme approche littéraire», in Nouveaux regards sur le texte littéraire, éd. par Vincent Jouve, Reims, Epure, 2013, p. 203-222.

Heidmann, Ute, Adam, Jean-Michel, Textualité et intertextualité des contes, Paris, Classiques Garnier, 2010.

Humboldt, Wilhelm von, "Ueber die Verschiedenheiten des menschlichen Sprachbaues» (1827-1829), in Werke, Darmstadt, WBG, 2010, vol. 3, p. 144-190.

Jakobson, Roman, Essais de linguistique générale, Paris, Editions de Minuit, 1963.

Labov, William, Le parler ordinaire, Paris, Editions de Minuit, 1978.

Laplantine, Chloé, "La langue de Baudelaire", une culturologie», Semen, 33 (2012), p. 71-90.

Maingueneau, Dominique, Philippe, Gilles, Exercices de linguistique pour le texte littéraire, Paris, Dunod, 1997.

Malinowski, Bronislaw, Les jardins de corail, Paris, La Découverte, 2002 (1965).

Meschonnic, Henri, Pour la poétique, Paris, Gallimard, 1970.

—, Poétique du traduire, Paris, Verdier, 1999.

—, Langage, histoire. Une même théorie, Paris, Verdier, 2012.

Monte, Michèle, Philippe, Gilles (éds), Genres et textes. Déterminations, évolutions, confrontations, Lyon, Presses universitaires de Lyon, 2013.

Moretti, Franco, La letteratura vista da lontano, Torino, Einaudi, 2005. —, Graphes, cartes et arbres. Modèles abstraits pour une autre histoire de la littérature, Paris, Les Prairies ordinaires, 2008 (2005).

NøLKE, Henning, «Linguistique modulaire: principes méthodologiques et applications", in Approches modulaires: de la langue au discours, éd. par Henning Nølke, Jean-Michel Adam, Paris/Lausanne, Delachaux et Niestlé, 2000, p. 17-73.

Pêcheux, Michel, Analyse automatique du discours, Paris, Dunod, 1969.

Propp, Vladimir, Morphologie du conte, Paris, Seuil, 1970 (1928/1969).

Rastier, François, Arts et sciences du texte, Paris, PUF, 2001.

Ricceur, Paul, Du texte à l'action, Paris, Seuil, 1986.

Roulet, Daniel de, "Johann Schneider-Ammann en pharaon", Le Temps, $1^{\text {er }}$ novembre 2012.

Roulet, Eddy, "Une approche modulaire de la complexité de l'organisation du discours", in Approches modulaires: de la langue au 
discours, éd. par Henning Nølke, Jean-Michel Adam, Paris/ Lausanne, Delachaux et Niestlé, 2000, p. 187-279.

Ruwet, Nicolas, "Parallélismes et déviations en poésie», in Langue, discours, société. Pour Emile Benveniste, éd. par Julia Kristeva, JeanClaude Milner, Nicolas Ruwet, Paris, Seuil, 1975, p. 307-351.

Schaeffer, Jean-Marie, Petite écologie des études littéraires, Paris, Thierry Marchaisse, 2011.

Schleiermacher, Friedrich Daniel Ernst, Herméneutique, Paris/Lille, Cerf/PUL, 1987 (1805-1833).

Slakta, Denis, "Esquisse d'une théorie lexico-sémantique: pour une analyse d'un texte politique (Cahiers de doléances)", Langages, 23 (1971), p. 87-134.

Starobinski, Jean, La relation critique, Paris, Gallimard, 1970.

—, "Leo Spitzer et la lecture stylistique», préface des Etudes de style (1970), Paris, Gallimard, 2001.

Todorov, Tzvetan, "La notion de littérature», in Langue, discours, société. Pour Emile Benveniste, éd. par Julia Kristeva, Jean-Claude Milner, Nicolas Ruwet, Paris, Seuil, 1975, p. 352-364.

—, Les genres de discours, Paris, Seuil, 1978.

—, Mikhaïl Bakhtine, le principe dialogique, Paris, Seuil, 1981.

Volochinov, Valentin Nikolaevich, Marxisme et philosophie du langage, Limoges, Lambert-Lucas, 2010 (1929).

Weinrich, Harald, Tempus, Stuttgart, Kohlhammer, 1994 (1964).

Wismann, Heinz, Penser entre les langues, Paris, Albin Michel, 2012.

Zambano, Francine, «Il faut renforcer les universités sans toucher à leur autonomie", entretien avec J. Schneider-Ammann, L'uniscope, 581 (2013), p. 16-17.

Zima, Peter Václáv, Théorie critique du discours, Paris, L'Harmattan, 2003. 
